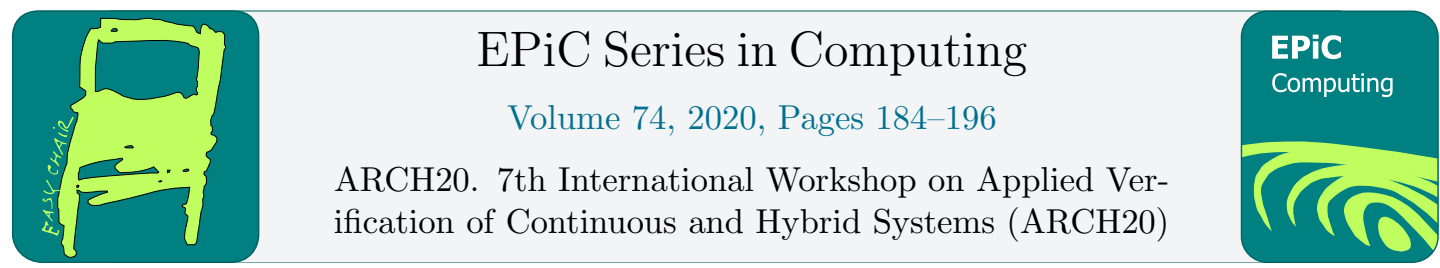

\title{
Kaa: A Python Implementation of Reachable Set Computation Using Bernstein Polynomials
}

\author{
Edward Kim and Parasara Sridhar Duggirala \\ Department of Computer Science \\ University of North Carolina at Chapel Hill \\ $\{$ ehkim,psd\}@cs.unc.edu
}

\begin{abstract}
Reachable set computation is one of the many widely-used techniques for the verification of safety properties of dynamical systems. One of the simplest algorithms for computing reachable sets for discrete nonlinear systems uses parallelotope bundles and Bernstein polynomials. In this paper, we describe Kaa, a terse Python implementation of reachable set computation which leverages the widely used symbolic package sympy. Additionally, we simplify the user interface and provide easy-to-use plotting utilities. We believe that our tool has pedagogical value given the simplicity of the implementation and its userfriendliness.
\end{abstract}

\section{Introduction}

Reachable set computation is one of the important tools for verification of safety properties of dynamical and hybrid systems. A simpler and easier-to-understand reachable set computation algorithm that utilizes Bernstein polynomials and parallelotopes has been presented in [4]. Several cumulative improvements $[5,15,3,7,8]$ have been proposed to improve its accuracy and efficiency. The tool SAPO [6] which implements these algorithms in C++ is available publicly. In this paper, we present a Python implementation of algorithm presented in [8]. Our motivation for reimplementing the algorithms in Python is two-fold. First, using standard libraries for symbolic manipulation in Sympy, the algorithm for reachability can be implemented concisely. In fact, the main algorithm for computing the reachable set has be implemented in only one file with 150 lines of code. The total code base for Kaa is roughly only 650 lines of code. In contrast, SAPO consists of more than 2000 lines of C++ code with memory and pointer management performed completely by the developer. We believe that such a simple implementation can be used for teaching one of the main algorithms for reachability of nonlinear systems. Second, reimplementing the tool in Python makes user interaction significantly simpler. In SAPO, one has to recompile the code along with the model file to generate the binary. Subsequently the user has to execute the binary to compute the reachable set. Additionally, visualizing the reachable set using SAPO is a two step process. In the first step, SAPO generates a MATLAB script which plots projections of reachable set for visualizing time or phase plots of the reachable set. In the second step, the user feeds the generated script into either MATLAB or GNU 
Octave to illustrate the reachable set. In contrast, Kaa offers plotting functionalities through the matplotlib library for visualizing the reachable set. The models and the code for computing reachable set using Kaa are provided in Python and do not require any compilation steps. Integrating these two, we provide an easy-to-use Jupyter notebook interface for computing and visualizing reachable set with Kaa for some of the standard benchmark models.

Bernstein polynomials are also an active area of research in the domain of global optimization [14, 10, 13]. Several heuristics have been proposed for improving the performance of optimization using Bernstein polynomials [1, 16, 12]. Having an accessible and flexible tool would make it more conducive to implement these heuristics and determine if they are helpful in the domain of reachability. In light of these features and advantages, we believe Kaa could be used as a natural first step for introducing reachability in a pedagogical setting.

\section{Preliminaries}

The state of a system, denoted as $x$, lies in a domain $D \subseteq \mathbb{R}^{n}$. A discrete-time polynomial nonlinear system is denoted as

$$
x^{+}=f(x)
$$

where $f(x): \mathbb{R}^{n} \rightarrow \mathbb{R}^{n}$ is polynomial in $x$. The trajectory of the system that evolves according to Equation (1), denoted as $\xi\left(x_{0}\right)$, is the sequence $x_{0}, x_{1}, \ldots$ where $x_{i+1}=f\left(x_{i}\right)$. The $k^{t h}$ element in this sequence is denoted as $x_{k}=\xi\left(x_{0}, k\right)$. Given an initial set $\Theta$, the reachable set at time $k$, denoted as $\Theta_{k}=\left\{\xi\left(x_{0}, k\right) \mid x_{0} \in \Theta\right\}$.

A parallelotope $P$ is a set of states in $\mathbb{R}^{n}$ denoted as $\langle\Lambda, c\rangle$ where $\Lambda \in \mathbb{R}^{2 n \times n}$ and $c \in \mathbb{R}^{2 n}$, $\Lambda_{i+n}=-\Lambda_{i}$ and $i \in\{1, \ldots, n\}$ such that

$$
x \in P \text { if and only if } \Lambda x \leq c .
$$

$\Lambda$ is called the direction matrix $\Lambda_{i}$ denotes the $i^{\text {th }}$ row of $\Lambda . c$ is called the offset. Alternatively, a parallelotope can also be represented in vertex-generator representation as $\left\langle v, g_{1}, \ldots, g_{n}\right\rangle$. Here $v \in \mathbb{R}^{n}$ is called vertex and $g_{1}, \ldots, g_{n}, g_{i} \in \mathbb{R}^{n}$, are called generators. The parallelotope is defined as

$$
P \triangleq\left\{x \mid \exists \alpha_{1}, \ldots, \alpha_{n}, x=v+\alpha_{1} g_{1}+\ldots+\alpha_{n} g_{n}, 0 \leq \alpha_{i} \leq 1\right\}
$$

This representation is very similar to Zonotopes [11, 2] and Star sets [9]. Notice that for a parallelotope $P$, the vertex-generator representation also defines the affine transformation that maps $[0,1]^{n}$ to $P$. We denote this affine transformation as $T_{p}$. A parallelotope bundle $Q$ is a set of parallelotopes $\left\{P_{1}, \ldots, P_{m}\right\}$ where $Q=\cap_{i=1}^{m} P_{i}$.

Given two multi-indices $i$ and $d$ of size $n$, where $i \leq d$, the Bernstein polynomial of degree $d$ and index $i$ is

$$
\mathcal{B}_{i, d}=\beta_{i_{1}, d_{1}}\left(x_{1}\right) \beta_{i_{2}, d_{2}}\left(x_{2}\right) \ldots \beta_{i_{n}, d_{n}}\left(x_{n}\right) .
$$

where $\beta_{i_{m}, d_{m}}\left(x_{m}\right)=\left(\begin{array}{c}d_{m} \\ i_{m}\end{array}\right) x_{m}^{i_{m}}\left(1-x_{m}\right)^{d_{m}-i_{m}}$. Any polynomial function can be expressed in the Bernstein basis. The primary advantage of the Bernstein representation of a polynomial $h\left(x_{1}, \ldots, x_{n}\right)$ is that an upper bound on the supremum and lower bound on the infimum of $h\left(x_{1}, \ldots, x_{n}\right)$ in $[0,1]^{n}$ can be computed purely by observing the coefficients of the polynomial in the Bernstein basis. 
In other words, given a polynomial $h\left(x_{1}, \ldots, x_{n}\right)=\sum_{j \in J} a_{j} \mathbf{x}_{j}$ where $J$ is a set of multiindices iterating through the degrees found in $p$ with $a_{j} \in \mathbb{R}$, then $h\left(x_{1}, \ldots, x_{n}\right)$ can be converted into its counterpart under the Bernstein basis, $h\left(x_{1}, \ldots, x_{n}\right)=\sum_{j \in J} b_{j} \mathcal{B}_{j}$ where $b_{j}$ are the corresponding Bernstein coefficients. The upper and lower bounds of $h\left(x_{1}, \ldots, x_{n}\right)$ over $[0,1]^{n}$ are bounded by the Bernstein coefficients:

$$
\min \left\{b_{1}, \ldots, b_{m}\right\} \leq \inf f_{x \in[0,1]^{n}} h(x) \leq \sup _{x \in[0,1]^{n}} h(x) \leq \max \left\{b, \ldots, b_{m}\right\} .
$$

As mentioned earlier, a parallelotope $P$ can also be represented as an affine transformation $T_{p}$ from $[0,1]^{n}$ to $P$. Therefore, upper bounds on the suprenum of a function $h$ over $P$ is equivalent to upper bound of $h \circ T_{p}$ over $[0,1]^{n}$. A similar argument follows for the lower bound on the infimum.

For the remainder of the document, we assume that by using functional composition and the Bernstein representation, we can compute the upper bound on supremum and the lower bound on the infimum of polynomial functions over parallelotopes. We denote the procedures for calculating such upper and lower bounds for a polynomial $h$ over some parallelotope $P$ as BernsteinUpper $(h, P)$ and BernsteinLower $(h, P)$ respectively.

\section{Reachability of Nonlinear Systems Using Parallelotope Bundles and Bernstein Polynomials}

Given a set represented as a parallelotope bundle $Q=\left\{P_{1}, P_{2}, \ldots, P_{m}\right\}$ and a discrete dynamical system $x^{+}=f(x)$, we now present the method for computing an overapproximation of the image $f(Q)$ as a new parallelotope bundle $Q^{\prime}=\left\{P_{1}^{\prime}, P_{2}^{\prime}, \ldots, P_{m}^{\prime}\right\}$. We ensure that direction matrix of $P_{i}^{\prime}$ is same as $P_{i}$ and the computation is required only to compute the offsets. Let us denote the $j^{\text {th }}$ offset of $P_{i}$ and $P_{i}^{\prime}$ as $c_{j, i}$ and $c_{j, i}^{\prime}$ respectively and the $j^{\text {th }}$ direction in $P_{i}$ (same as $P_{i}^{\prime}$ ) as $\Lambda_{j, i}$. Therefore, given $\Lambda$, transformation $f$, and offsets $c_{j, i}$ for $P_{i}$, we have to compute the values of $c_{j, i}^{\prime}$.

If $j \leq n$, any offset $c_{j, i}^{\prime}$ such that $\forall_{x \in Q} \Lambda_{j, i} \cdot f(x) \leq c_{j, i}^{\prime}$ is valid. If $j>n$, then any offset $c_{j, i}^{\prime}$ such that $\forall_{x \in Q} \Lambda_{j-n, i} \cdot f(x) \geq c_{j, i}^{\prime}$ is valid. Therefore, one can compute the $j^{\text {th }}$ offset of $P_{i}^{\prime}$, i.e., $c_{j, i}^{\prime}$ for $j \leq n$ by computing an upper bound of the function $\Lambda_{j, i} \cdot f(x)$ over the $x \in P_{i}$. Similarly, the $j+n^{t h}$ offset can be computed from a lower bound of the function $\Lambda_{j, i} \cdot f(x)$ over each parallelotope $x \in P_{i}$. This is given in Equations 2 and 3 .

$$
\begin{aligned}
c_{j, i} & =\min _{l=1}^{m}\left\{\operatorname{BernsteinUpper}\left(\Lambda_{j, i} \cdot f(x), P_{l}\right)\right\} \text { if } j \leq n . \\
c_{j+n, i} & =\max _{l=1}^{m}\left\{\operatorname{BernsteinLower}\left(\Lambda_{j, i} \cdot f(x), P_{l}\right)\right\} \text { otherwise. }
\end{aligned}
$$

\subsection{Python Implementation of Reachable Set Computation}

One of the primary reasons for preferring Python as the implementation language for this algorithm is the presence of powerful, well-tested symbolic and matrix-computation libraries. Python's numpy libraries are popular matrix-computation libraries which allow higher-level manipulation of matrices. This gives us an avenue of overcoming the verbosity and the possibility of memory leaks inherent in implementing identical features in $\mathrm{C}++$. We believe that 
overcoming these obstacles results in a more readable, more compact approach to parallelotope reachability suitable for graduate students and curious practitioners. In addition, these libraries are known for their extensive set of useful functionalities which allow us to avoid reimplementing many fundamental operations.

As we have seen before, there are two main sub-routines for computing the reachable set using Bernstein polynomials. First, performing functional composition for computing the upper bound of a polynomial over a parallelotope. Second, computing the Bernstein representation of a polynomial. The library of sympy has powerful symbolic manipulation tools which allow us to comfortably perform many sensitive symbolic function composition of polynomials. We use sympy's internal representation of polynomials to (a) perform functional composition and (b) compute the Bernstein representations of the resulting polynomial. Additionally, the matplotlib library has accessible plotting facilities that we integrate into our tool for visualizing the reachable set. In particular, matplotlib facilitates the ability to plot several reachable sets simultaneously. This will aid others in performing more complex bundle-transformation experiments in the future.

Finally, Python contains a rich set of multiprocessing libraries, namely multiprocessing or $m p$ for short. We plan to exploit the parallelizable nature of bundle-transformtion computations using $m p$ 's ability to provide powerful multiprocessing features without much verbosity.

\section{Evaluations}

We evaluate our tool on the benchmarks that are shipped along with SAPO. With each benchmark, the reachable sets of SAPO are juxtaposed with that of Kaa's to demonstrate the accuracy and quality of our reachable sets. A comparison of time taken by Kaa and SAPO for each benchmark is provided in Table 3 .

\subsection{SIR Epidemic Model}

The SIR Epidemic model is a 3-dimensional dynamical system governed by the following dynamics:

$$
\begin{aligned}
s_{k+1} & =s_{k}-\left(\beta s_{k} i_{k}\right) \Delta \\
i_{k+1} & =i_{k}+\left(\beta s_{k} i_{k}-\gamma i_{k}\right) \Delta \\
r_{k+1} & =r_{k}+\left(\gamma i_{k}\right) \Delta
\end{aligned}
$$

where $s, i, r$ represent the fractions of a population of individuals designated as susceptible, infected, and recovered respectively. There are two parameters, namely $\beta$ and $\gamma$, which influence the evolution of the system. $\beta$ is labeled as the contraction rate and $1 / \gamma$ is mean infective period. Finally, $\Delta$ is simply the discretization step. For the benchmarks, we set $\beta=0.34, \gamma=0.05$, and $\Delta=0.5$. The reachable sets are displayed in Figure 1 . The table of numerical value comparisions between Sapo and Kaa is shown in Table 1.

Here, offu is the vector of upper offsets of the parallelotope and offl is the vector for the lower offsets. We define the upper facets of parallelotope $P$ as the $c_{i}$ for $i \leq n$ where $n$ is the dimension of the system and $P=\langle\Lambda, c\rangle$ is the parallelotope. Similarly, the lower facets are defined as the $c_{i+n}$ for $i \leq n$. In the case above, we are looking at values in $c_{2}$ and $c_{2+3}=c_{5}$. 


\begin{tabular}{|c|cccc|}
\hline Time Steps & Kaa (offu) & Sapo (offu) & Kaa (off) & Kaa (off) \\
\hline 50 & 0.470716 & 0.470716 & -0.435191 & -0.43519 \\
51 & 0.475839 & 0.475839 & -0.439599 & -0.439599 \\
52 & 0.480906 & 0.480906 & -0.443945 & -0.443945 \\
53 & 0.485915 & 0.485915 & -0.448227 & -0.448227 \\
54 & 0.490862 & 0.490862 & -0.452443 & -0.452443 \\
55 & 0.495747 & 0.495747 & -0.456591 & -0.456591 \\
56 & 0.500566 & 0.500566 & -0.460669 & -0.460669 \\
57 & 0.505317 & 0.505317 & -0.464675 & -0.464675 \\
58 & 0.509999 & 0.509999 & -0.4686075 & -0.468608 \\
59 & 0.514610 & 0.514610 & -0.472465 & -0.472465 \\
60 & 0.519147 & 0.519147 & -0.476246 & -0.476246 \\
\hline
\end{tabular}

Table 1: Comparision of offu, offl values along variable $i$ for the SIR model. We select the steps $50-60$

\subsection{Rossler Model}

The Rossler model is another 3-dimensional system governed under the dynamics:

$$
\begin{aligned}
& x_{k+1}=x_{k}+-(y-z) \Delta \\
& y_{k+1}=y_{k}+\left(x_{k}+a y_{k}\right) \Delta \\
& z_{k+1}=z_{k}+\left(b+z_{k}\left(x_{k}-c\right)\right) \Delta
\end{aligned}
$$

where $a, b, c$ are parameters which we set to $a=0,1, b=0.1$, and $c=14$. We set our discretization step to be $\Delta=0.025$. The reachable sets are displayed in Figure 2 and the table showing the comparisions between the numerical values are shown in Table 2.

\begin{tabular}{|c|cccc|}
\hline Time Steps & Kaa (offu) & Sapo $(\mathbf{o f f u})$ & Kaa (off) & Kaa (off) \\
\hline 50 & 1.95908 & 1.96043 & -1.9209 & -1.9193 \\
51 & 1.83552 & 1.83688 & -1.7963 & -1.7947 \\
52 & 1.71044 & 1.71181 & -1.67016 & -1.6685 \\
53 & 1.58392 & 1.58531 & -1.54255 & -1.5409 \\
54 & 1.45604 & 1.45744 & -1.41355 & -1.4119 \\
55 & 1.32687 & 1.32829 & -1.28324 & -1.2815 \\
56 & 1.19649 & 1.19792 & -1.15168 & -1.1500 \\
57 & 1.06499 & 1.06642 & -1.01896 & -1.0172 \\
58 & 0.932432 & 0.933877 & -0.885157 & -0.88339 \\
59 & 0.798905 & 0.800358 & -0.75035 & -0.74857 \\
60 & 0.664489 & 0.665949 & -0.614619 & -0.61282 \\
\hline
\end{tabular}

Table 2: Comparision of offu, offl values along variable $y$ for the Rossler model. We select the steps $50-60$ 


\subsection{Quadcopter Model}

The Quadcopter model is a 17-dimensional dynamical system with the following variables: A set of inertial positions: $\left(p_{n}, p_{e}, h\right)$, linear velocities $(u, v, w)$, the quaternions expressing the Euler angles $\left(q_{0}, q_{1}, q_{2}, q_{3}\right)$, the angular velocities $(p, q, r)$, and finally the parameters $\left(h_{I}, u_{I}, v_{I}, \psi_{I}\right)$. The complete set of dynamics and relevant parameters is found in [8] and reachable sets are shown in Figure 3.

\subsection{Lotka-Volterra Model}

We also test on the competetitive Lotka-Volterra models for predator-prey biological systems. Our instance is a 5-dimensional system governed as below:

$$
\begin{aligned}
x_{k+1} & =x_{k}+\left(x_{k}\left(1-\left(x_{k}+\alpha y_{k}+\beta \ell_{k}\right)\right)\right) \Delta \\
y_{k+1} & =y_{k}+\left(y_{k}\left(1-\left(y_{k}+\alpha z_{k}+\beta x_{k}\right)\right)\right) \Delta \\
z_{k+1} & =z_{k}+\left(z_{k}\left(1-\left(z_{k}+\alpha h_{k}+\beta y_{k}\right)\right)\right) \Delta \\
h_{k+1} & =h_{k}+\left(h_{k}\left(1-\left(h_{k}+\alpha \ell_{k}+\beta z_{k}\right)\right)\right) \Delta \\
\ell_{k+1} & =\ell_{k}+\left(\ell_{k}\left(1-\left(\ell_{k}+\alpha x_{k}+\beta h_{k}\right)\right)\right) \Delta
\end{aligned}
$$

where $\alpha, \beta$ are parameters set to $\alpha=0.85$ and $\beta=0.5$. We set $\Delta=0.01$. The relevant reachable sets are shown in Figure 4.

\subsection{Phosphoraley Model}

The Phosphoraley model describes a certain cellular regulatory system. It is captured by seven variables governed by the following dynamics:

$$
\begin{aligned}
x_{k+1}^{1} & =x_{k}^{1}+\left(-\alpha x_{k}^{1}+\beta x_{k}^{3} x_{k}^{4}\right) \Delta \\
x_{k+1}^{2} & =x_{k}^{2}+\left(\alpha x_{k}^{1}-x_{k}^{2}\right) \Delta \\
x_{k+1}^{3} & =x_{k}^{3}+\left(x_{k}^{2} \beta x_{k}^{3} x_{k}^{4}\right) \Delta \\
x_{k+1}^{4} & =x_{k}^{4}+\left(\beta x_{k}^{5} x_{k}^{6}-\beta x_{k}^{3} x_{k}^{4}\right) \Delta \\
x_{k+1}^{5} & =x_{k}^{5}+\left(-\beta x_{k}^{5} x_{k}^{6}+\beta x_{k}^{3} x_{k}^{4}\right) \Delta \\
x_{k+1}^{6} & =x_{k}^{6}+\left(\alpha x_{k}^{7}-\beta x_{k}^{5} x_{k}^{6}\right) \Delta \\
x_{k+1}^{7} & =x_{k}^{7}+\left(-\alpha x_{k}^{7}+\beta x_{k}^{5} x_{k}^{6}\right) \Delta
\end{aligned}
$$

where $\alpha, \beta$ are two parameters assigned as $\alpha=0.5$ and $\beta=5$. We set $\Delta=0.01$ here. The figures can be found in Figure 5 .

\subsection{Times}

It is evident from Table 3 that while the current implementation in Python is intuitive and concise, it incurs severe performance penalties. Nevertheless, we pursued this direction because of the pedagogical value in making this an accessible tool for implementing and understanding reachability. To this end, the repository contains a Jupyter notebook with the name kaa-intro designed to interactively introduce graduate students and practitioners to the usage of Kaa. The notebook even contains a subset of the examples shown above, giving the auidence a hands-on method to run the code for themselves. An immediate next step is to deploy extensive profiling to find performance bottlenecks and subsequently improve on them. 
Table 3: Reachable Set Computation Time of Benchmarks

\begin{tabular}{|l|cc|}
\hline Model & Kaa & SAPO $(\mathrm{C}++)$ \\
\hline SIR & $11.41 \mathrm{sec}$ & $0.16 \mathrm{sec}$ \\
Rossler & $41.92 \mathrm{sec}$ & $1.17 \mathrm{sec}$ \\
Quadcopter & $78.21 \mathrm{sec}$ & $11.98 \mathrm{sec}$ \\
Lotka-Volterra & $18 \mathrm{~min} 95.05 \mathrm{sec}$ & $57.48 \mathrm{sec}$ \\
Phosphoraley & $103.81 \mathrm{sec}$ & $24.86 \mathrm{sec}$ \\
\hline
\end{tabular}

\section{Conclusions}

We present Kaa, a Python implementation of reachable set computation of nonlinear systems which is focused towards accessibility and pedagogical use. The usage of inbuilt sympy libraries makes the implementation short and simple (only $650 \mathrm{LOC}$ ). While we do incur performance drawbacks from selecting Python for implementing this algorithm, we believe that it aids in fast prototyping and enables students to easily build on top of the library. In particular, Python's readability and extendibility will allow curious students to experiment with more involved bundle-transformations. The code can be accessed through https:

//github.com/Tarheel-Formal-Methods/kaa.

Acknowledgements: This material is based upon work supported by the Air Force Office of Scientific Research under award number FA9550-19-1-0288 and National Science Foundation (NSF) under grant numbers CNS 1739936, 1935724. Any opinions, findings, and conclusions or recommendations expressed in this material are those of the author(s) and do not necessarily reflect the views of the United States Air Force or National Science Foundation.

\section{References}

[1] Kodiak, a C++ library for rigorous branch and bound computation, https://github.com/nasa/ Kodiak, Accessed: July 2020

[2] Althoff, M., Stursberg, O., Buss, M.: Computing reachable sets of hybrid systems using a combination of zonotopes and polytopes. Nonlinear analysis: hybrid systems 4(2), 233-249 (2010)

[3] Dang, T., Dreossi, T., Piazza, C.: Parameter synthesis using parallelotopic enclosure and applications to epidemic models. In: International Workshop on Hybrid Systems Biology. pp. 67-82. Springer (2014)

[4] Dang, T., Salinas, D.: Image computation for polynomial dynamical systems using the bernstein expansion. In: International Conference on Computer Aided Verification. pp. 219-232. Springer (2009)

[5] Dang, T., Testylier, R.: Reachability analysis for polynomial dynamical systems using the bernstein expansion. Reliable Computing 17(2), 128-152 (2012)

[6] Dreossi, T.: Sapo: Reachability computation and parameter synthesis of polynomial dynamical systems. In: Proceedings of the 20th International Conference on Hybrid Systems: Computation and Control. pp. 29-34 (2017)

[7] Dreossi, T., Dang, T., Piazza, C.: Parallelotope bundles for polynomial reachability. In: Proceedings of the 19th International Conference on Hybrid Systems: Computation and Control. pp. 297-306 (2016)

[8] Dreossi, T., Dang, T., Piazza, C.: Reachability computation for polynomial dynamical systems. Formal Methods in System Design 50(1), 1-38 (2017) 
[9] Duggirala, P.S., Viswanathan, M.: Parsimonious, simulation based verification of linear systems. In: International Conference on Computer Aided Verification. pp. 477-494. Springer (2016)

[10] Garloff, J.: The bernstein expansion and its applications. Journal of the American Romanian Academy 25, 27 (2003)

[11] Girard, A.: Reachability of uncertain linear systems using zonotopes. In: International Workshop on Hybrid Systems: Computation and Control. pp. 291-305. Springer (2005)

[12] Muñoz, C., Narkawicz, A.: Formalization of bernstein polynomials and applications to global optimization. Journal of Automated Reasoning 51(2), 151-196 (2013)

[13] Nataraj, P.S., Arounassalame, M.: A new subdivision algorithm for the bernstein polynomial approach to global optimization. International journal of automation and computing 4(4), 342352 (2007)

[14] Nataray, P., Kotecha, K.: An algorithm for global optimization using the taylor-bernstein form as inclusion function. Journal of Global Optimization 24(4), 417-436 (2002)

[15] Sassi, M.A.B., Testylier, R., Dang, T., Girard, A.: Reachability analysis of polynomial systems using linear programming relaxations. In: International Symposium on Automated Technology for Verification and Analysis. pp. 137-151. Springer (2012)

[16] Smith, A.P.: Fast construction of constant bound functions for sparse polynomials. Journal of Global Optimization 43(2-3), 445-458 (2009) 

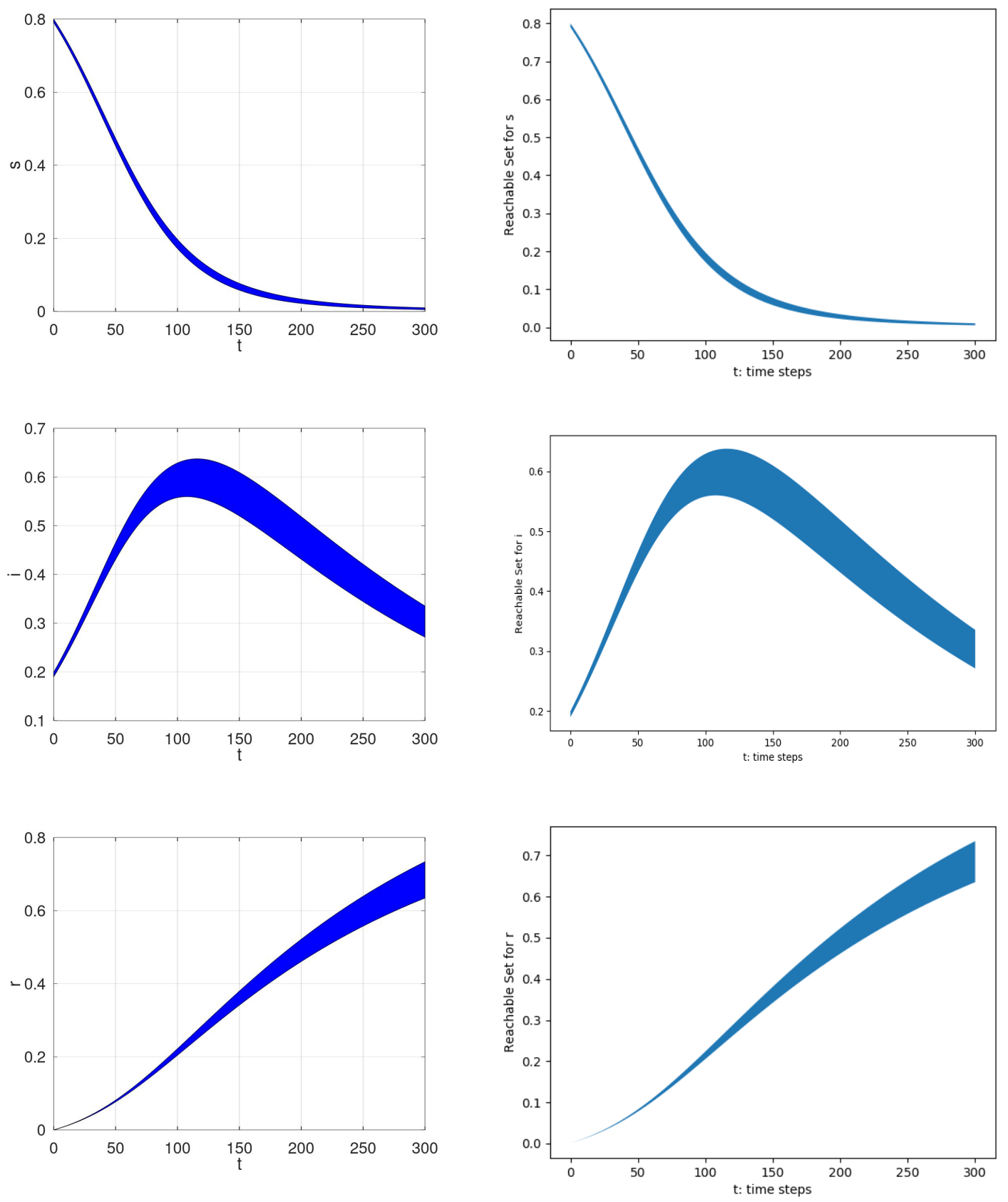

Figure 1: Figure depicting the reachable set computation of the SIR model. 

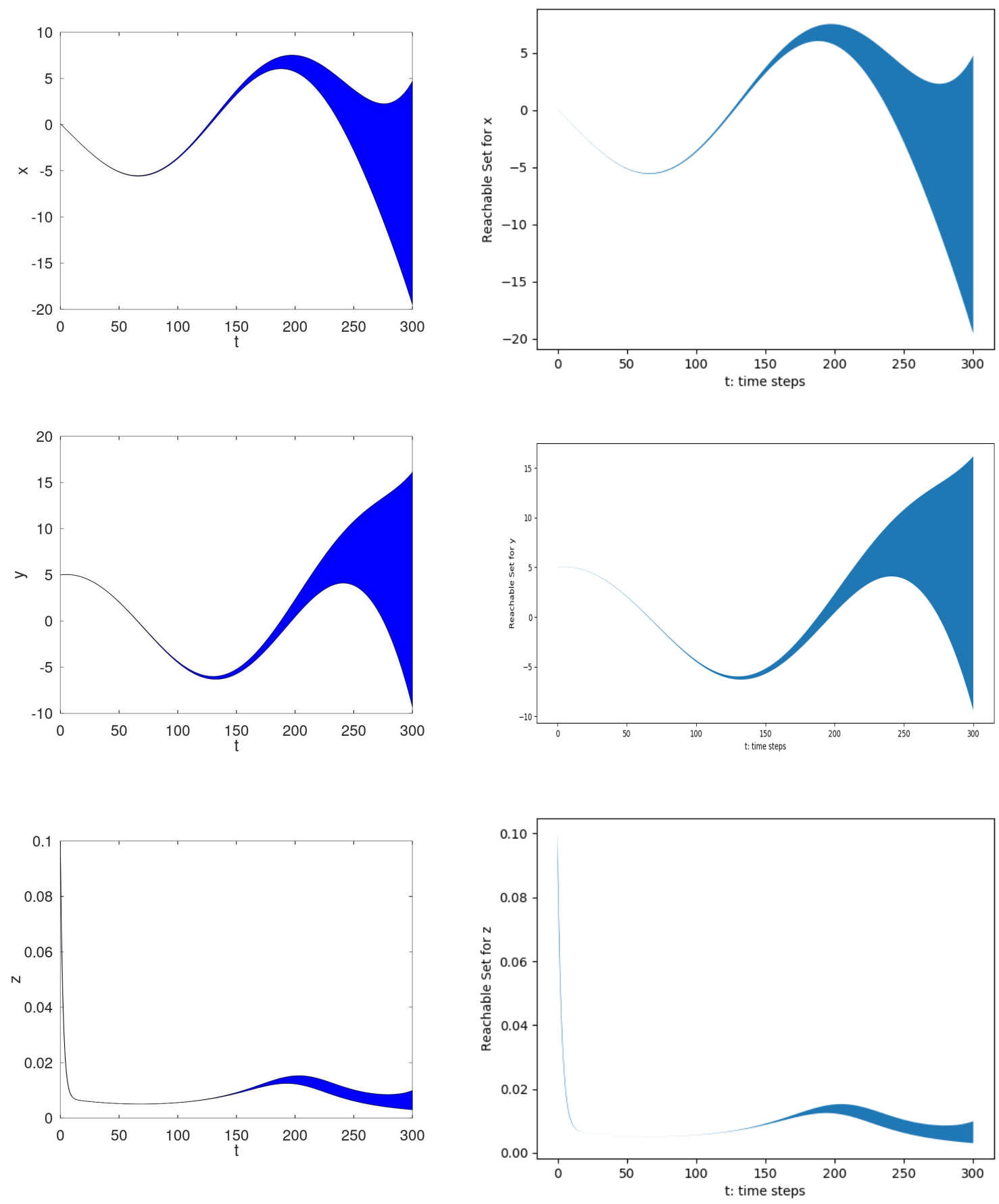

Figure 2: Figure depicting the reachable set computation of the Rossler model. 

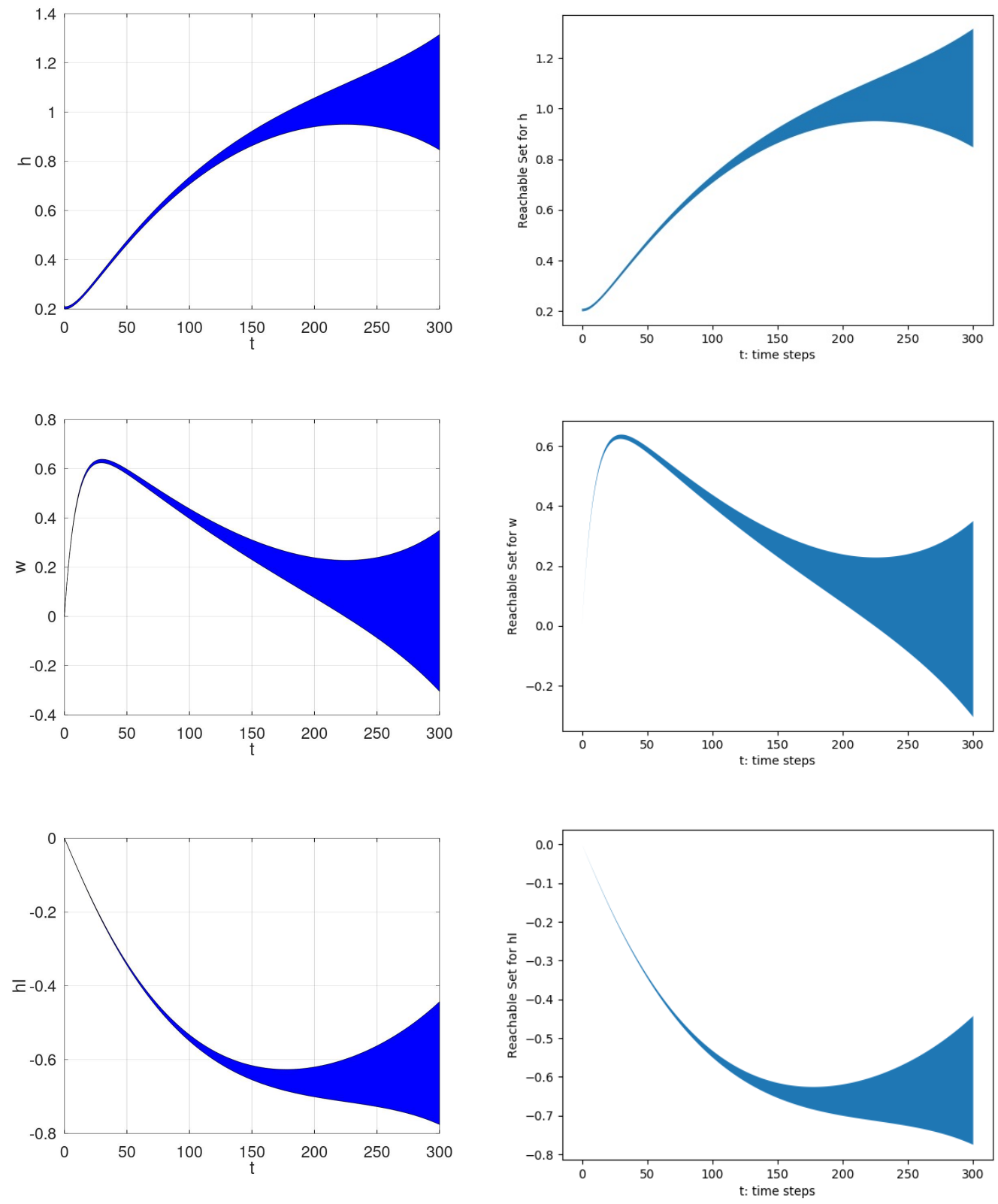

Figure 3: Figure depicting the reachable set computation of the Quadcopter model. 

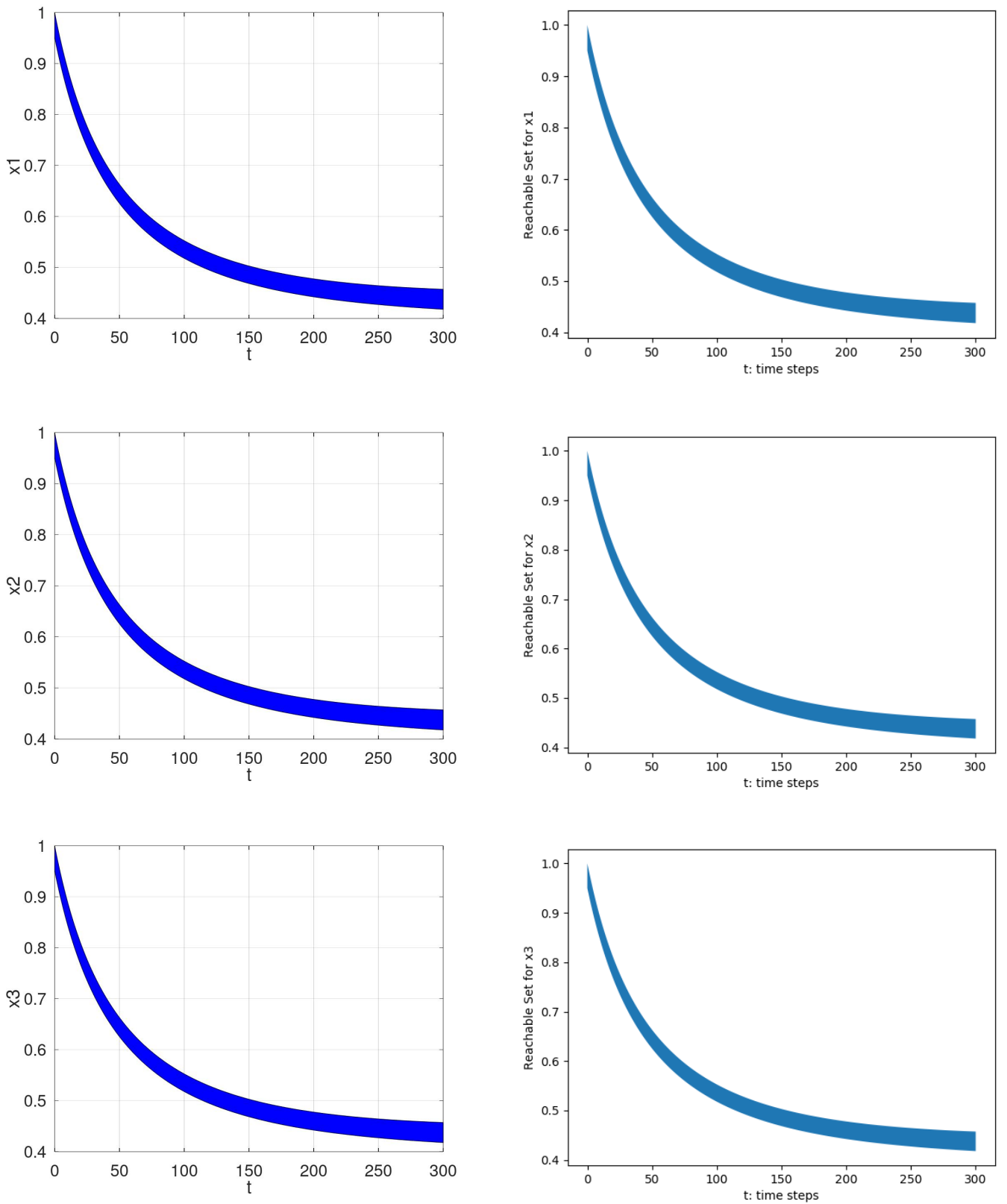

Figure 4: Figure depicting the reachable set computation of the Lotka-Volterra model. 

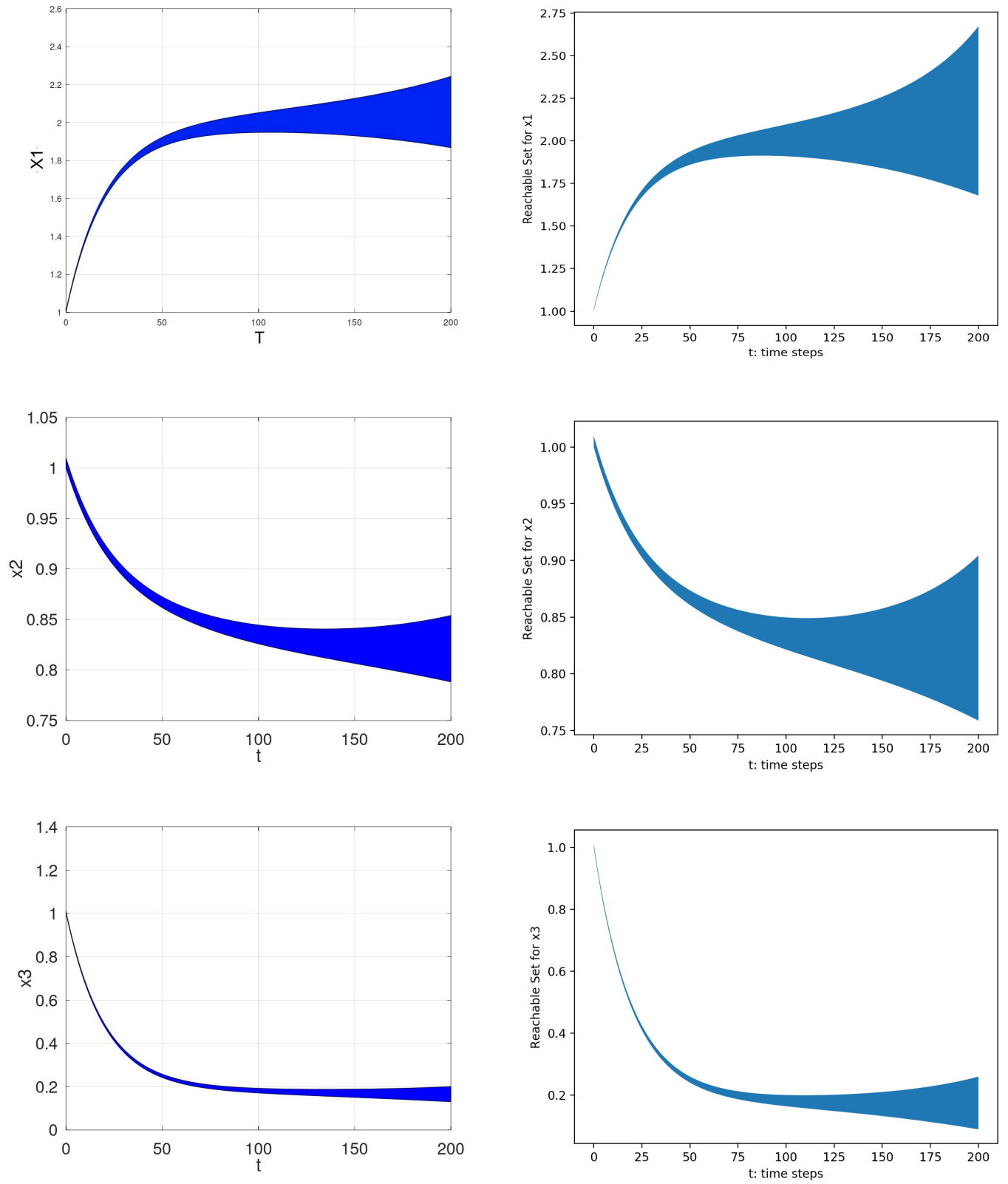

Figure 5: Figure depicting the reachable set computation of the Phosporaley model. 